\title{
Ablación endovenosa por radiofrecuencia para el tratamiento de las várices. Revisión sistemática
}

\author{
Endovenous radiofrequency ablation for the treatment of \\ varicose veins. Systematic review
}

\section{Ablação por radiofreqüência endovenosa para o tratamento de veias varicosas. Revisão sistemática}

doi http://dx.doi.org/10.35954/SM2020.39.2.4

\author{
Martín Alvez a (D) https://orcid.org/0000-0003-2168-3245 \\ José Cabillón b (D) https://orcid.org/0000-0002-8834-6465
}
(a) Jefe de Servicio de Cirugía Vascular. Hospital Central de las Fuerzas Armadas. Ex Prof. Adj. de Cirugía Vascular titular. Mutualista COSEM y Sociedad Médica Universal. Cirujano Vascular de Trasplante Hepático. Hospital Central de las Fuerzas Armadas.

(b) Cirujano General del Servicio de Cirugía General. Hospital Central de las Fuerzas Armadas.

Ex Asistente de Clínica Quirúrgica. Clínica Quirúrgica "A", Facultad de Medicina, Universidad de la República. Cirujano Titular. Mutualista Servicio Médico Integral.

\section{RESUMEN}

La insuficiencia venosa de los miembros inferiores ha sido motivo de estudio y controversia dada la alta prevalencia que presenta. En los últimos años hubo un avance significativo en el diagnóstico y tratamiento de esta patología con el surgimiento de múltiples procedimientos mínimamente invasivos. De este modo, sumado al tratamiento quirúrgico clásico, se describen la inyección de espuma, la radiofrecuencia y el endoláser. Estas últimas técnicas, tienen la ventaja de menores complicaciones, menos dolor postoperatorio, así como mejores resultados estéticos. Todos ellos requieren una técnica quirúrgica depurada lo que implica una curva de aprendizaje exponencial, así como la utilización sistemática de ecodoppler.

Se realizó una revisión sistemática del tema analizando la bibliografía publicada en los últimos 20 años en nuestro país y el mundo. Comparando las diferentes técnicas miniinvasivas, la radiofrecuencia ha demostrado ventajas, con mejores tasas de recuperación, reintegro laboral mas rápido, menores complicaciones, así como buenos resultados estéticos, siendo una técnica segura y altamente efectiva. Por todo esto puede ser considerada hoy como una de las primeras líneas de tratamiento en el paciente afecto de varices.

PALABRAS CLAVE: Ablación por Radiofrecuencia; Insuficiencia Venosa; Procedimientos Endovasculares; Terapia por Láser; Várices. 


\section{ABSTRACT}

Venous insufficiency of the lower limbs has been the subject of study and controversy given its high prevalence. In recent years there has been a significant advance in the diagnosis and treatment of this pathology with the emergence of multiple minimally invasive procedures.

In this way, in addition to the classical surgical treatment, foam injection, radiofrequency and the endolaser are described. These last techniques have the advantage of fewer complications, less postoperative pain, as well as better cosmetic results. All of them require a refined surgical technique, which implies an exponential learning curve, as well as the systematic use of Doppler ultrasound.

A systematic review of the subject was carried out, analyzing the bibliography published in the last 20 years in our country and the world. Comparing the different minimally invasive techniques, radiofrequency has shown advantages, with better recovery rates, faster return to work, fewer complications as well as good aesthetic results, being a safe and highly effective technique. For all this it can be considered today as one of the first lines of treatment in patients with varicose veins.

KEY WORDS: Radiofrequency ablation; Venous Insufficiency; Endovascular Procedures; Laser Therapy; Varicose Veins.

\section{RESUMO}

A insuficiência venosa dos membros inferiores tem sido um tema de estudo e controvérsia devido a sua alta prevalência. Nos últimos anos, houve um avanço significativo no diagnóstico e tratamento desta patologia com o surgimento de múltiplos procedimentos minimamente invasivos.

Assim, além do tratamento cirúrgico clássico, são descritas a injeção de espuma, a radiofrequência e o endolaser. Estas últimas técnicas têm a vantagem de menos complicações, menos dores pós-operatórias, assim como melhores resultados estéticos. Todas elas exigem uma técnica cirúrgica refinada que implica uma curva de aprendizagem exponencial, bem como o uso sistemático do echo-Doppler.

Foi realizada uma revisão sistemática do assunto, analisando a bibliografia publicada nos últimos 20 anos em nosso país e no mundo.

Comparando as diferentes técnicas mini-invasivas, a radiofreqüência tem mostrado vantagens, com melhores taxas de recuperação, retorno mais rápido ao trabalho, menos complicações, bem como bons resultados estéticos, sendo uma técnica segura e altamente eficaz. Por todas estas razões, ela pode ser considerada hoje como uma das primeiras linhas de tratamento no paciente afetado por varizes.

PALAVRAS CHAVE: Ablação por Radiofrequência; Insuficiência Venosa; Procedimentos Endovasculares; Terapia a Laser; Varizes.

\section{INTRODUCCIÓN}

La insuficiencia venosa crónica tiene una elevada prevalencia a nivel mundial (1). El origen de ésta, en la mayoría de los casos, se da a nivel del sistema superficial (safeno interno y/o externo) secundario a una insuficiencia valvular y reflujo. Esto conlleva a la hipertensión venosa, generando la aparición de várices y trastornos tróficos (2).
El tratamiento clásico de la insuficiencia venosa superficial a nivel de los troncos safenos ha sido la safenectomía y resección de las colaterales varicosas (3).

Esta técnica mantiene su vigencia actual, pero como desventaja principal en comparación con otros tratamientos, es el mayor dolor postoperatorio por la aparición de equímosis y hematomas. Además, se han descrito otras complicaciones 
como el dolor neurítico por trauma del nervio safeno, infecciones y malos resultados estéticos (4). Existen otras técnicas menos invasivas de amplio uso en otros medios, como ser la ablación endoluminal térmica (láser o radiofrecuencia), inyección ecoguiada de esclerosantes con espuma y últimamente la obliteración con pegamento (cianoacrilato) (5). El objetivo de la ablación por radiofrecuencia es generar una oclusión fibrótica de la vena patológica y su consecuente atrofia, mediante la transmisión de calor a través de una sonda endoluminal (6).

\section{OBJETIVOS}

El objetivo general de este trabajo es realizar una revisión sistemática del tema, analizando las indicaciones de la terapia, describir una técnica novedosa en nuestro país y efectuar un análisis comparativo con otros métodos de tratamiento.

\section{MATERIALES Y MÉTODOS}

Se realizó una búsqueda bibliográfica en Medline/ PubMed, The Cochrane Library y EMBASE utilizando varias combinaciones de lenguaje natural y controlado. Los idiomas utilizados fueron el español, inglés y portugués, siendo las palabras clave "Várices", "Procedimientos endovasculares", "Técnicas de ablación", "Insuficiencia venosa". Los criterios de inclusión fueron: artículos sobre el tratamiento endovascular de las várices publicadas en inglés o español desde 1994 hasta la fecha.

\section{DESARROLLO DEL TEMA}

La insuficiencia venosa crónica (IVC) de los miembros inferiores constituye un grave problema de salud pública con una gran repercusión socioeconómica, debido a su elevada prevalencia e incidencia, estando presente en hasta el $50 \%$ de las personas (7).

La insuficiencia venosa se define como aquella situacióńn fisiopatológica caracterizada por la incapacidad del sistema venoso para mantener un flujo anterógrado. Entre las alteraciones funcionales se destacan dos elementos: la insuficiencia valvular y la hipertensión venosa (8).
Clínicamente podrán o no ser sintomáticas, e incluyen una amplia gama de signos clínicos que varían desde una mínima dilatación venosa superficial hasta cambios crónicos, IVC, siendo su máxima expresión la ulceración. El síntoma más prevalente es el dolor, que se describe como pesadez de toda la extremidad. El edema el segundo síntoma más prevalente se describe por los pacientes como la hinchazón de las extremidades inferiores que aumenta a lo largo del día. Se manifiesta con carácter progresivo de distal a proximal (9). Cambios tróficos en la piel. El enlentecimiento de la circulación venosa da oportunidad al filtrado de la hemosiderina que progresivamente va tatuando la piel, que toma un tinte color pardo. Esta hiperpigmentación se alterna con áreas de color blanquecino de piel atrófica. La inflamación progresiva y engrosamiento de la piel a causa de la fibrosis de la dermis y el tejido celular subcutáneo (lipodermatoesclerosis). No es infrecuente observar la asociación de descamación en algunas áreas que en ocasiones actúan como pequeñas puertas de entrada de infecciones. En casos de insuficiencia venosa avanzada la existencia de una úlcera abierta o ya cicatrizada completa el cuadro clínico (10).

\section{EXAMEN FÍSICO}

El paciente con patología venosa se debe inspeccionar específicamente en bipedestación, con el fin de lograr un relleno venoso máximo.

La inspección revelará abultamientos de las venas superficiales en el territorio afecto, con dilatación de sus colaterales según la afectación dependa de la safena interna, externa o ambas.

Algunos casos menos comunes presentan dilataciones en territorios más atípicos como la cara posterior del muslo, con dependencia de perforantes de dicho sector.

La inspección también revela las áreas de hiperpigmentación, atrofia blanca lipodermatoesclerosis o úlceras. Las úlceras han de describirse en relación a su localización, típicamente perimaleolares, (donde mayor presión hidrostática se produce en proximidad a las venas perforantes) tamaño, fondo, exudado y sus características (10). 


\begin{tabular}{|c|c|c|c|}
\hline \multicolumn{4}{|c|}{ Clasificación CEAP de la EVC } \\
\hline \multirow{7}{*}{ Clínica } & Clase 0 & No signos visibles & \multirow{7}{*}{$\begin{array}{l}\text { Asintomático } \\
\text { Sintomático }\end{array}$} \\
\hline & Clase 1 & Telangiectasias o varices reticulares & \\
\hline & Clase 2 & Varices & \\
\hline & Clase 3 & Edema & \\
\hline & Clase 4 & Signos dérmicos & \\
\hline & Clase 5 & Úlcera cicatrizada & \\
\hline & Clase 6 & Úlcera activa & \\
\hline Etiología & Congénita & Primaria & Secundaria \\
\hline Anatomía & Superficial y/o & Profunda & Perforante \\
\hline Patofisiología & Reflujo y/o & Obstrucción & \\
\hline
\end{tabular}

Tabla 1. Clasificación CEAP (11).

Para facilitar la comunicación y la descripción de la IVC se creó la clasificación CEAP, acrónimo de Clínica, Etiología, Anatomía y Patofisiología (tabla 1). Esta clasificación, posee la ventaja de estandarizar y estudiar todos los trastornos, empleando el mismo lenguaje para diferentes países, facilitando el análisis de las diferentes opciones terapéuticas, que en el caso de la patología venosa alcanzan hoy día un amplio espectro, con el consiguiente beneficio a la hora de reconocer el seguimiento evolutivo, los resultados terapéuticos y profundizar en la historia natural de la Insuficiencia Venosa Crónica (11).

A pesar de sus limitaciones, que se han intentado minimizar con sus actualizaciones, es la más empleada hoy día. Existiendo inclusive una aplicación basada en la misma en Apple Store.

La última actualización fue realizada en 2020 en la que se realizaron los siguientes cambios: se agregaron las clases clínicas $\mathrm{C} 2 \mathrm{r}$ y C6r para consignar la presencia de várices y úlcera recurrente respectivamente, la clase 4 se subdivide en tres, C4a pigmentación, C4b lipodermatoesclerosis o atrofia blanca y C4c corona flebectática. En cuanto a la clasificación etiológica, actualmente se discrimina a la patología venosa secundaria en intravenosa o extravenosa (Esi-Ese). La anatomía se consigna mediante la abreviatura de los respetivos nombres de las venas implicadas y a la fisiopatología se le agrega la categoríaa $\mathrm{Pn}$ (sin patología identificada) para describir aquellos pacientes que no presentan reflujo ni obstrucción pero aún así muestran dilataciones venosas y/o alteraciones hemodinámicas (12).

\section{DIAGNÓSTICO}

\section{Ecodoppler}

El ecodoppler o duplex, es recomendado como el primer estudio diagnóstico para todo paciente con sospecha de insuficiencia venosa crónica. Este test es seguro, no invasivo, costo-efectivo y confiable. Permite detectar obstrucción, turbulencia y dirección del flujo venoso y arterial. Es excelente para la evaluación de obstrucciones infrainguinales e incompetencia valvular a este nivel. Para un examen dúplex completo se deben valorar las características anatómicas, compresibilidad y flujo venosos incluyendo la medición de la duración del reflujo (13).

El estudio debe ser realizado de forma sistematizada con el paciente de pie y en decúbito, dependiendo la estructura a analizar (figura 1). Se deben obtener imágenes transversales y longitudinales. La visión transversal otorga información general precisa en cuanto a morfología y la posible presencia de trombo endoluminal (con maniobras compresivas), mientras que la visión longitudinal permite evaluar la presencia de reflujo y la cuantificación del mismo (14).

Cuando se plantea la ablación por radiofrecuencia de la vena safena interna, es esencial evaluar su permeabilidad, tortuosidad, su continuidad intrafascial, su diámetro, la presencia de duplicaciones o grandes tributarias. Su uso intraoperatorio es además imprescindible para la visualización del catéter, por lo que el cirujano debe estar familiarizado con el mismo (15). 


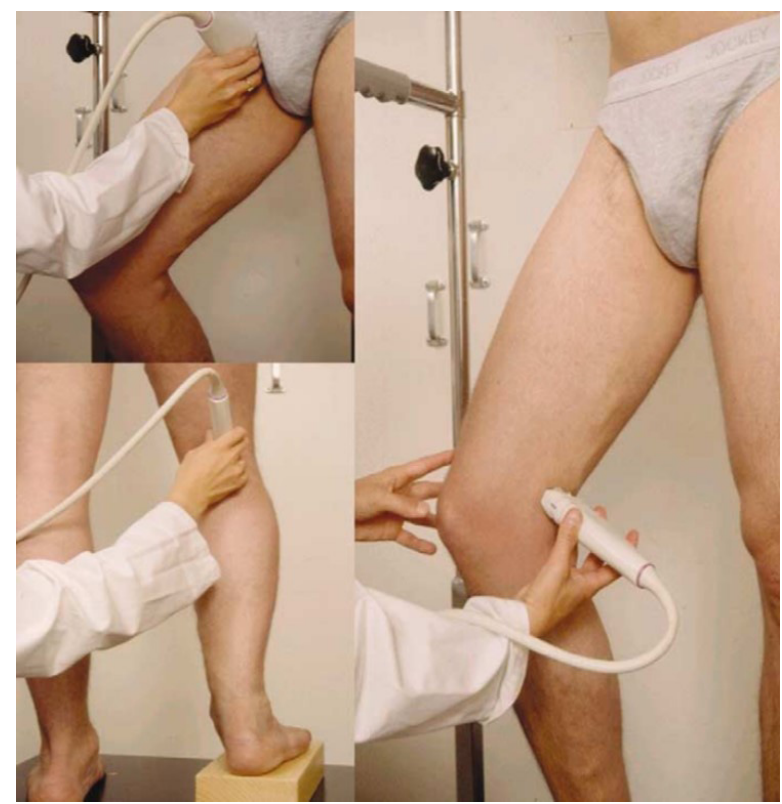

Figura 1. Posición del paciente durante el estudio con ecodoppler.

\section{Ecodoppler}

El manejo de la insuficiencia venosa inicia con medidas higiénico-dietéticas, asociadas al uso de terapia compresiva y eventualmente farmacológica. Hoy en día, no se concibe el tratamiento quirúrgico, como una simple ablación completa del sistema venoso superficial, y el abordaje terapéutico de la enfermedad varicosa no consiste en extirpar mayor o menor cantidad de venas. Se trata de una delicada intervención capaz de cumplir con los siguientes requisitos: eliminar todos los puntos de reflujo y resecar aquellos segmentos de las venas superficiales enfermos, debido a un fallo valvular, el cual identificamos en forma selectiva mediante el ecodoppler color. Además, siempre debe realizarse la operación de las várices pensando en el mejor resultado estético (16).

La cirugía ha demostrado a lo largo de muchos años su utilidad en el tratamiento de la insuficiencia venosa, con buenos resultados en cuanto a tasas de recidiva y confort postoperatorio (4). El stripping consiste en la ligadura de la unión proximal de la safena mayor o menor, fleboextracción de la vena safena, y flebectomía de los lagos venosos insuficientes y de las venas tributarias.
En las últimas décadas han surgido diferentes técnicas de tratamiento endovascular, con resultados comparables a la cirugía abierta (stripping) en cuanto a tasas de recidivas y menor dolor postoperatorio (13).

\section{PRINCIPIO Y MODO DE ACCIÓN DE LAS TÉCNICAS ENDOLUMINALES DE TERMOABLACIÓN}

\section{Radiofrecuencia}

Es un mecanismo por el cual un catéter introducido por vía endovenosa emite energía térmica por radiofrecuencia desde un generador. Su objetivo es provocar el espasmo venoso inducido por calor, la denudación endotelial y la disminución y contracción del colágeno de la pared venosa, elevando la temperatura entre 85 y $90{ }^{\circ} \mathrm{C}$. En la actualidad existe un nuevo catéter de acción segmentaria $(7 \mathrm{~cm})$, que eleva la temperatura a $120^{\circ} \mathrm{C}$ en forma controlada y liberando energía constante (máximo $20 \mathrm{~W}$ ) que no admite cambios por parte del usuario (17).

El procedimiento Closure Fast, elimina la necesidad de un abordaje de la ingle y la anestesia general, y por lo general se realiza con anestesia local en el consultorio de un especialista en la vena o en un centro quirúrgico ambulatorio. El procedimiento dura aproximadamente 45-60 minutos y la mayoría de los pacientes suelen pasar de dos a tres horas en el postoperatorio (18).

\section{Endoláser}

El mecanismo de acción del endoláser es diferente a la radiofrecuencia. En este caso se produce daño tisular intimal por la burbuja de vapor generada con temperaturas que llegan a ser de $800{ }^{\circ} \mathrm{C}$ en la punta de la fibra, lo que conduce a una reacción inflamatoria y posterior fibrosis (19). 


\section{Selección del paciente para termoablación con Radiofrecuencia}

Los pacientes susceptibles de tratamiento con esta técnica son aquellos con signos y síntomas de insuficiencia venosa y estudio mediante ecografía doppler color compatible con reflujo mayor a 0.5 segundos, sistema venoso profundo permeable y vena pasible de cateterizar (distancia a la piel $>1 \mathrm{~cm}$ ).

Los criterios de exclusión absolutos incluyen la trombosis venosa profunda aguda, la tromboflebitis superficial aguda, las infecciones en el sitio de punción, y la obstrucción del sistema venoso profundo si la vena a tratar es una colateral funcional. Los criterios de exclusión relativos son la tortuosidad venosa, venas menores de $2 \mathrm{~mm}$ o mayores de $25 \mathrm{~mm}$, la obstrucción parcial proximal de la vena, la existencia de trombofilia conocida, la enfermedad arterial periférica concomitante, la inmovilización y el embarazo (18).

\section{Técnica quirúrgica}

Para la realización del procedimiento (15) se precisa la utilización de un ecógrafo doppler color, tanto para la punción, como para observar el avance del catéter o la obliteración de la vena tratada. Los pasos de realización de la técnica son:

1. Mediante punción ecoguiada con el paciente en posición supina, se coloca un introductor de $7 \mathrm{Fr}$ en la vena safena interna, normalmente a nivel de la rodilla.

2. Se avanza la punta del catéter hasta colocarlo a $2 \mathrm{~cm}$ de la unión safeno-femoral. Dicho avance puede facilitarse mediante el empleo de una guía.

3. Mediante control ecográfico se realiza la infiltración/anestesia tumescencia en el compartimento fascial alrededor de la vena.

4. Se trata a la vez un segmento de $7 \mathrm{~cm}$ de vena durante un ciclo de tratamiento de 20 segundos, habitualmente a $120{ }^{\circ} \mathrm{C}$. Los siguientes segmentos se tratan de forma secuencial.
5. Las marcas en el cuerpo del catéter permiten recolocar el mismo de forma rápida y exacta entre ciclos de tratamiento.

6. El tratamiento de una vena de $45 \mathrm{~cm}$ tarda de 3 a 5 minutos (siete segmentos de tratamiento), puesto que el primero se hace doble.

7. Tras el tratamiento, las extremidades son envueltas mediante un vendaje compresivo y medias de compresión de clase II, insistiendo a los pacientes en la deambulación precoz. Luego de 24-72 horas, se retira el vendaje, continuando el empleo de las medias durante 2 semanas.

\section{DISCUSIÓN}

Si tenemos en cuenta la fisiopatología de la insuficiencia venosa crónica es evidente, que para obtener resultados eficaces y duraderos en el tratamiento de las varices es fundamental eliminar el origen del reflujo venoso $(20,21)$.

La información obtenida sobre la hemodinamia venosa procedente de los estudios mediante ecodoppler, demuestra que las varices de las extremidades inferiores son debidas en la mayoría; de los casos $(70 \%)$ a insuficiencia del cayado y/o troncular de la vena safena interna (VSI). Con menor frecuencia se pueden ver afectados otros sistemas como son la vena safena externa, venas perforantes y/o tributarias $(22,23)$.

Hasta fines de los años 90 el único tratamiento quirúrgico eficaz y duradero para tratar las varices tronculares ha sido la ligadura del cayado safeno-femoral y stripping de la VSI. Durante las últimas décadas, el progreso tecnológico ha permitido el desarrollo y aplicación de nuevas terapias mínimamente invasivas como la radiofrecuencia endovenosa y el endoláser (5).

La ablación de la vena safena interna por radiofrecuencia comenzó a utilizarse en Europa en el año 1998, y luego de su aprobación por la Food and Drug Administration (FDA) en 1999 en los Estados Unidos (17).

Desde el año 2000 hay publicaciones que demuestran experimentalmente la abolición del reflu- 
jo safeno con radiofrecuencia. El estudio multicéntrico publicado por Merchant y colaboradores con 1.222 extremidades tratadas y un seguimiento de 4 años, demostró éxito ecográfico y satisfacción del paciente en mas del $85 \%$ de los casos $(24,25)$. $\mathrm{Al}$ inicio se utilizaron catéteres de primera generación con los cuales no se obtuvo buenos resultados debido a su difícil manipulación y a las restricciones con venas de diámetros mayores a 12 $\mathrm{mm}$. Estos se caracterizaban por tener un manejo complejo y una velocidad de retiro o "pullback» muy lenta, lo que hacía que el procedimiento fuera prolongado. Por otro lado, existían 2 medidas de catéteres, de 6 y $8 \mathrm{Fr}$, para venas de hasta 8 y $12 \mathrm{~mm}$ de diámetro, respectivamente, siendo este último el diámetro máximo para tratar permitido por la FDA hasta ese momento.

En el año 2007 se lanzan al mercado una nueva generación de catéteres ClosureFast (Medtronic, Minneapolis, Minnesota, Estados Unidos) que debido a sus características físicas eran más fáciles de manipular, más rápido el procedimiento y se podían obtener buenos resultados en venas mayores de $12 \mathrm{~mm}$ con baja tasa de complicaciones. Así lo demuestra el trabajo realizado por Bauza Moreno y cols., en donde queda demostrado la reducción del diámetro a los 6 meses del procedimiento, siendo estadísticamente significativa, donde el diámetro máximo tratado fue de $25 \mathrm{~mm}$, con una reducción de este a los 6 meses del $56 \%$ (11 $\mathrm{mm})(26)$.

\section{Comparación de la técnica de radiofrecuencia con los otros métodos}

Para evaluar los resultados de las diferentes técnicas se utilizan hoy en día principalmente dos scores clínicos, el venous clinical severity score (VCSS) y el chronic venous insufficiency quality of life (CIVIQ).

El primero compara características de la severidad de la enfermedad venosa tales como dolor, varices, edema, pigmentación entre otros, divididos en ausente [0] leve [1] moderado [2] severo
[3] realizando así una escala para definir diferentes grupos de severidad.

El segundo es un cuestionario que se realiza en los pacientes con IVC MMII midiendo el impacto en su calidad de vida. Así por ejemplo evalúa el dolor durante el día, limitaciones en el trabajo y en sus actividades diarias.

Son varias las publicaciones de estudios comparativos de radiofrecuencia vs stripping.

Un estudio comparativo multicéntrico aleatorizado Endovenous Obliteration vs Ligation and Vein Stripping (EVOLVeS) demostró una recuperación y un reintegro laboral más rápido en los pacientes en que se realizó radiofrecuencia con un resultado estadísticamente significativo $(27,28)$.

La morbilidad (dolor, equimosis y hematomas) fue mayor en el grupo de cirugía convencional. La calidad de vida postoperatoria (CIVIQ2) fue significativamente mejor para los pacientes tratados con radiofrecuencia.

No hubo diferencias hemodinámicas en la valoración por ecodoppler al año y a los 2 años. Además, demostró menor dolor en reposo y bipedestación, con RFA fundamentalmente en postoperatorio inmediato con menor necesidad de analgésicos. También demostró una recuperación física y reintegro al trabajo más rápido. En los 3 años de seguimiento no hubo diferencia en la recidiva varicosa. La principal ventaja de la radiofrecuencia frente al endoláser es la trasmisión controlada del calor lo cual disminuye significativamente el riesgo de lesiones térmicas de los tejidos vecinos. Un estudio controlado randomizado a 5 años, comparó las diferentes técnicas de termoablación (radiofrecuencia y láser), escleroterapia con espuma y cirugía, demostrando un mayor índice de recanalización de la vena safena mayor con la escleroterapia, y sin diferencias significativas entre las otras terapias $(29,30)$. 


\section{CONCLUSIONES}

En este siglo hubo un avance importante en los tratamientos mínimamente invasivos en medicina en general, pero fundamentalmente en los procedimientos quirúrgicos.

La patología venosa no escapa a esta situación y se han descripto múltiples procedimientos, cada vez menos agresivos y con mejores resultados.

La ablación por radiofrecuencia endovenosa es una técnica segura y altamente efectiva que permite el tratamiento de las varices con elevada satisfacción del paciente, retorno precoz a la actividad normal y prácticamente ausencia de dolor. En la mayoría de los casos ha demostrado su superioridad frente a la cirugía convencional. Además, al ser indolora y ofrecer mejor calidad de vida frente al endoláser, puede ser considerada hoy como una de las primeras líneas de tratamiento del paciente afecto de varices.

En cuanto a las complicaciones, estas son muy infrecuentes, destacándose trombosis venosa, quemadura cutánea y neuritis, pero son fácilmente prevenibles cuando se realiza una adecuada y depurada técnica. Cabe destacar la utilización en la cirugía del ecodoppler, fundamental e imprescindible para planificar la estrategia, realizar un adecuado control de todos los pasos del procedimiento, así como el correcto seguimiento de los pacientes.

DECLARACIÓN DE CONFLICTOS DE INTERESES: Los autores no reportan ningún conflicto de interés. El estudio se realizó con recursos propios de los autores y/o la institución a la que representan.

\section{REFERENCIAS}

(1) Kaplan RM, Criqui MH, Denenberg JO, Bergan J, Fronek A. Quality of life in patients with chronic venous disease: San Diego population study. J Vasc Surg 2003; 37(5):1047-53.

doi: $10.1067 / m v a .2003 .168$
(2) Takase S, Pascarella L, Bergan JJ, Schmid-Schönbein GW.

Hypertension induced venous valve remodeling. J Vas Surg 2004; 39(6):1329-34.

doi: 10.1016/j.jvs.2004.02.044

(3) Winterborn RJ, Foy C, Earnshaw JJ.

Causes of varicose vein recurrence: late results of a randomized controlled trial of stripping the long saphenous vein.

J Vasc Surg 2004; 40(4):634-9.

doi: 10.1016/j.jvs.2004.07.003

(4) Murad MH, Coto-Yglesias F, Zumaeta-García M, Elamin MB, Duggirala MK, Erwin PJ, et al.

A systematic review and meta-analysis of the treatments of varicose veins.

J Vasc Surg 2011; 53(5 Suppl):49S-65S.

doi: 10.1016/j.jvs.2011.02.031

(5) Siribumrungwong B, Noorit P, Wilasrusmee C, Attia J, Thakkinstian A. A systematic review and meta-analysis of randomized controlled trials comparing endovenous ablation and surgical intervention in patients with varicose vein.

Eur J Vasc Endovasc Surg 2012; 44(2):214-23. doi: 10.1016/j.ejvs.2012.05.017

(6) Proebstle TM, Vago B, Alm J, Göckeritz O, Lebard C, Pichot O, et al. Treatment of the incompetent great saphenous vein by endovenous radiofrequency powered seg- mental thermal ablation: first clinical experience.

J Vasc Surg 2008; 47(1):151-156

doi: 10.1016/j.jvs.2007.08.056

(7) Chiesa R, Marone EM, Limoni C, Volontè M, Petrini O. Chronic venous disorders: correlation between visible signs, symptoms, and presence of functional disease.

J Vasc Surg 2007; 46(2):322-30.

doi:10.1016/j.jvs.2007.04.030

(8) Takase S, Pascarella L, Bergan JJ, Schmid-Schönbein GW.

Hypertension induced venous valve remodeling. J Vas Surg 2004; 39(6):1329-1334

doi: 10.1016/j.jvs.2004.02.044 
(9) Porter JM, Moneta GL. Reporting standards in venous disease: an update. International Consensus Committee on Chronic Venous Disease. J Vasc Surg 1995; 21(4):635-45.

doi: 10.1016/s0741-5214(95)70195-8

(10) Bradbury A, Evans C, Allan P, Lee A, Ruckley V, Fowkes FGR. What are the symptoms of varicose veins? Edinburgh vein study cross sectional population survey.

BMJ 1999; 318(7180):353-6.

doi: $10.1136 / \mathrm{bmj} .318 .7180 .353$

(11) Beebe HG, Bergan JJ, Bergqvist D, Eklöf B, Eriksson, I, Goldman MP, et al. Classification and grading of chronic venous disease in the lower limbs: a consensus statement. Eur J Vasc Endovasc Surg 1996; 12(4):487-91; discussion 491-2. doi:10.1016/s1078-5884(96)80019-0

(12) Lurie F, Passman M, Meisner M, Dalsing M, Masuda E, Welch $\mathrm{H}$, et al. CEAP classification system and reporting standard, revision 2020. J Vasc Surg Venous Lymphat Disord 2020; 8(3):342-352. doi: 10.1016/j.jvsv.2019.12.075

(13) Gloviczki P, Comerota AJ, Dalsing MC, Eklof BG, Gillespie DL, et al. The care of patients with varicose veins and associated chronic venous diseases: Clinical practice guidelines of the Society for Vascular Surgery and the American Venous Forum. J Vasc Surg 2011; 53(5 Suppl):2S-48S. doi: 10.1016/j.jvs.2011.01.079

(14) Coleridge-Smith P, Labropoulos N, Partsch H, Myers K, Nicolaides A, Cavezzi A.

Duplex Ultrasound Investigation of the Veins in Chronic Venous Disease of the Lower Limbs--UIP Consensus Document. Part I. Basic Principles.

Eur J Vasc Endovasc Surg 2006; 31(1):83-92. doi: 10.1016/j.ejvs.2005.07.019

(15) Roth SM. Endovenous Radiofrequency Ablation of Superficial and Perforator Veins.

Surg Clin North Am 2007; 87(5):1267-84, xii. doi: 10.1016/j.suc.2007.07.009
(16) De Maeseneer M, Pichot O, Cavezzi A, Earnshaw J, van Rij A, Lurie F, et al.

Duplex ultrasound investigation of the veins of the lower limbs after treatment for varicose veins - UIP consensus document.

Eur J Vasc Endovasc Surg 2011; 42(1):89-102. doi: 10.1016/j.ejvs.2011.03.013

(17) García Madrid C, Pastor Manrique JO, Arcediano Sanchez V, Sala-Planell E.

Endovenous radiofrequency ablation (VenefitTM procedure): Impact of different energy rates on great saphenous vein shrinkage.

Ann Vasc Surg 2013; 27(3):314-21.

doi: 10.1016/j.avsg.2012.06.015

(18) Zuniga JMR, Hingorani A, Ascher E, Shiferson A, Jung D, Jimenez R, et al.

Short-term outcome analysis of radiofrequency ablation using ClosurePlus vs ClosureFast catheter in the treatment of incompetent great saphenous vein. J Vasc Surg 2012; 55(4):1048-51.

doi: 10.1016/j.jvs.2011.11.050

(19) Schmedt CG, Sroka R, Steckmeier S, Meissner OA, Babaryka G, Hunger K, et al. Investigation on Radiofrequency and Laser $(980 \mathrm{~nm})$ Effectsafter Endoluminal Treatment of Saphenous Vein Insufficiencyin an Ex-vivo Model. Eur J Vasc Endovasc Surg 2006; 32(3):318-25. doi: 10.1016/j.ejvs.2006.04.013

(20) Dwerryhouse S, Davies B, Harradine K, Earnshaw JJ. Stripping the long saphenous vein reduces the rate of reoperation for recurrent varicose veins: five-year results of a randomized trial. J Vasc Surg 1999; 29(4):589-92.

doi: 10.1016/s0741-5214(99)70302-2

(21) Winterborn RJ, Foy C, Earnshaw JJ.

Causes of varicose vein recurrence: late results of a randomized controlled trial of stripping the long saphenous vein.

J Vasc Surg 2004; 40(4):634-9.

doi: 10.1016/j.jvs.2004.07.003 
(22) Labropoulos N, Leon M, Nicolaides AN, Giannoukas AD, Volteas N, Chan P. Superficial venous insufficiency: correlation of anatomic extent of reflux with clinical symptoms and signs. J Vasc Surg 1994; 20(6):953-8.

doi: 10.1016/0741-5214(94)90233-x

(23) Labropoulos N, Delis K, Nicolaides AN, Leon M, Ramaswami G. The role of the distribution and anatomic extent of reflux in the development of signs and symptoms in chronic venous insufficiency. J Vasc Surg 1996; 23(10)9:504-10. doi: 10.1016/s0741-5214(96)80018-8

(24) Merchant RF, De Palma RG, Kabnick LS. Endovascular obliteration of saphenous reflux: a multicenter study. J Vasc Surg 2002; 35(6):1190-6. doi: $10.1067 / m v a .2002 .124231$

(25) Merchant RF, Pichot O, Closure Study Group. Long-term outcomes of endovenous radiofrequency obliteration of saphenous reflux as a treatment for superficial venous insufficiency.

J Vasc Surg 2005; 42(3):502-9.

doi: 10.1016/j.jvs.2005.05.007

(26) Bauzá Moreno H, Dotta M, Katsini R, Marquez Fosser C, Rochet S, Pared C, et al. Ablación endovascular por radiofrecuencia. Efecto sobre el diámetro venoso con el uso del catéter ClosureFast ${ }^{\circledR}$.

Cir Esp 2016; 94(6):353-357.

doi: 10.1016/j.ciresp.2016.02.008

(27) Lurie F, Creton D, Eklof B, Kabnick LS, Kistner RL, Pichot O, et al. Prospective randomized study of endovenous radiofrequency obliteration (closure procedure) versus ligation and stripping in a selected patient population (EVOLVeS Study). $J$ Vasc Surg 2003; 38(2):207-14.

doi: 10.1016/s0741-5214(03)00228-3

(28) Lurie F, Creton D, Eklof B, Kabnick LS, Kistner RL, Pichot O, et al. Prospective randomised study of endovenous radiofrequency obliteration (closure) versus ligation and vein stripping (EVOLVeS): two-year follow-up.

Eur J Vasc Endovasc Surg 2005; 29(1):67-73.

doi: 10.1016/j.ejvs.2004.09.019
(29) Rautio T, Ohinmaa A, Perälä J, Ohtonen P, Heikkinen $\mathrm{T}$, Wiik $\mathrm{H}$, et al. Endovenous obliteration versus conventional stripping operation in the treatment of primary varicose veins: a randomized controlled trial with comparison of the costs. J Vasc Surg 2002; 35(5):958-65. doi: 10.1067/mva.2002.123096

(30) Lawaetz M, Serup J, lawaetz B, Bjoern L, Blemings $\mathrm{A}$, Eklof $\mathrm{B}$, et al. Comparison of endovenous ablation techniques, foam sclerotherapy and surgical stripping for great saphenous varicose veins. Extended 5-year follow-up of a RCT. Int Angiol 2017; 36(3)281-8.

doi: $10.23736 /$ S0392-9590.17.03827-5 